\title{
Mechanisms of carrier transport in CdTe polycrystalline films
}

\author{
A.V. Sukach, V.V. Tetyorkin and N.M. Krolevec \\ V. Lashkaryov Institute of Semiconductor Physics, NAS of Ukraine, \\ 41, prospect Nauky, 03028 Kyiv, Ukraine \\ Phone: 38 (044) 525-18-13, e-mail: teterkin@isp.kiev.ua
}

\begin{abstract}
Cadmium telluride (CdTe) polycrystalline films grown on glass ceramic substrates by a modified close-spaced vapor transport technique have been investigated. The as-grown films have columnar structure with the average grain sizes about $10 \mu \mathrm{m}$. The in-plain direct current conductivity as a function of temperature and electric field has been investigated. The percolation conductivity is shown to be dominant at low temperatures $(T<250 \mathrm{~K})$. At room temperature, the dominant transport mechanism is an activated process such as thermionic emission. The carrier transport across barriers is influenced by traps in the surface barrier regions. The non-annealed films exhibited stable electrical parameters and high photosensitivity during five-year storage under laboratory conditions.
\end{abstract}

Keywords: CdTe, polycrystalline film, percolation conductivity.

Manuscript received 19.02.10; accepted for publication 25.03.10; published online 30.04.10.

\section{Introduction}

Cadmium telluride (CdTe) is recognized as a promising photovoltaic material for diverse optoelectronic applications [1]. Due to its near-optimum direct energy gap (1.5 eV at room temperature) and high absorption coefficient, it allows a high theoretical maximum efficiency of $29 \%$ under AM1.5G conditions for CdTebased solar cells [2]. Recently $\mathrm{CdS} / \mathrm{CdTe}$ thin-film solar cells with efficiencies over $16.4 \%$ [3] and module performance over $11 \%$ [4] were demonstrated. In addition, CdTe has suitable physical properties like high atomic number and high density for utilization in X-ray and $\gamma$-ray detection $[5,6]$. There is growing interest to digital $\mathrm{X}$-ray images for obtaining and processing digital images in a real time regime. Polycrystalline $\mathrm{CdTe}$ as a material with high density and high stopping power can be successfully used for this purpose as well.

The key problems of $\mathrm{CdS} / \mathrm{CdTe}$ solar cells such as high series resistance, formation of ohmic contacts, degradation of solar cell performance are closely connected with conduction mechanisms in CdTe polycrystalline films. At the same time, the conductivity in these films has not been investigated systematically so far. Obviously, the lack of fundamental scientific knowledge of transport properties of CdTe polycrystalline films impedes further progress towards improving $\mathrm{CdS} / \mathrm{CdTe}$ solar cells performance.

Usually, polycrystalline semiconductors are regarded as having two different phases. One of them is the crystalline material inside a grain. Its physical properties is assumed to be similar to bulk material. The other one is the intergrain material, that is the grain boundaries (GBs). Accordingly, there are two conduction channels in polycrystalline materials connected with grains and GBs [7-9]. The conductivity in the first channel is controlled by potential barriers at the GBs. The grain boundary conductivity was investigated in $\mathrm{ZnO}$ varistors [9]. Information on similar investigations in other semiconductors is rather poor because of crystalline structure and electronic properties of GBs are not well understood so far. They were considered to be either between ordered crystal and disordered amorphous material $[7,8]$. In CdTe polycrystalline films of $n$-and $p$-type conductivity, GBs were modeled by $n-p-n$ structure [7] and dielectric layer [10].

It is generally accepted that the direct current conductivity in polycrystalline semiconductors is controlled by potential barriers and space charge regions at GBs [7,8]. There are two distinct mechanisms whereby mobile carriers can overcome a potential barrier at a grain boundary: quantum-mechanical tunneling and barrier emission. Earlier studies have shown that if the conductivity of a polycrystalline semiconductor exhibits Arrhenius behavior, then the thermally excited conduction mechanism like thermionic emission is dominant [7, 8, 11]. Frequently observed deviations from this plot can be attributed to fluctuations of potential barriers or to structural and electronic 
inhomogeneity of GBs [11]. If the scatter of the potential barrier heights is large enough, the direct current conductivity of a polycrystalline semiconductor is percolation in its nature [12]. An attempt to study the percolation conductivity in a sample of bulk polycrystalline $\mathrm{CdTe}$ has been performed previously [13].

The aim of this work is to study electrical and photoelectrical properties of thin CdTe polycrystalline films grown on dielectric substrates in a wide range of voltage biases and temperatures in order to clarify mechanisms of the direct current conductivity.

\section{Preparation of samples and experimental details}

Preparation of CdTe polycrystalline films with stable and predictable characteristics still to be an actual task for device applications. Different kinds of vapor deposition techniques [1] have been used for growing CdTe polycrystalline films on semiconductor and dielectric substrates. Among others, the close-spaced vapor transport technique possesses several advantages [14]. Since the as-grown films have poor electrical properties, they are usually annealed in $\mathrm{CdCl}_{2}$ atmosphere.

In this study, CdTe polycrystalline films were prepared by a close-spaced vapor transport technique modified in our laboratory. Samples of mechanically polished glass ceramic were used as substrates. The glass ceramic possesses such advantages as manufacturability, high transparency in a wide spectral range, thermal and chemical stability. The substrates were etched in aqueous solution of hydrofluoric acid and washed in distilled water. For deposition, polycrystalline CdTe of $n$-type conductivity doped with indium was used as a source. The substrate holder and source container were done from a high-density graphite block. Both have individual heater which allows one to regulate their temperatures with the accuracy $\pm 3{ }^{\circ} \mathrm{C}$. The distance $h$ between the substrate and the surface of CdTe source was adjusted by one to three millimeters. The developed technological module was placed in a quartz ampoule and mounted in a vacuum chamber $\left(P=10^{-4} \mathrm{~Pa}\right)$ using vertical arrangement.

The source and substrate temperatures $\left(T_{\mathrm{so}}, T_{\mathrm{sub}}\right)$ as well as the distance $h$ were varied in order to determine optimal deposition conditions. Directly before deposition, the substrate was heated to approximately $600{ }^{\circ} \mathrm{C}$ and maintained at this temperature during a halfhour. After that, the substrate temperature was gradually decreased up to the required value $350-400{ }^{\circ} \mathrm{C}$. Simultaneously, the source temperature was increased to values $500-600{ }^{\circ} \mathrm{C}$. The films investigated in this study had the mean grain size $9 \pm 2 \mu \mathrm{m}$ and resistance $\rho=(2$ $3) \times 10^{5} \mathrm{Ohm} \cdot \mathrm{cm}$. They were grown for approximately $15 \mathrm{~min}$ at $T_{\text {sub }}=400{ }^{\circ} \mathrm{C}$ and $T_{\text {so }}=550{ }^{\circ} \mathrm{C}$.

The as-prepared films were of $n$-type conductivity. The type of conductivity was checked by measuring the polarity of photoresponse signal in Schottky contacts deposited on the films. Previously, the polarity of photoresponse signal was calibrated using a sample of CdTe film with the known type of conductivity. The Schottky contacts were prepared by electrolyte deposition of Au. Ohmic contacts were deposited by thermal vacuum evaporation of indium. The measurements of the direct current conductivity were made using the standard four-probe technique. The distance between potential electrodes was within the range 1-2 $\mathrm{mm}$.

It is necessary to note that the investigated films were not annealed. They exhibited high photosensitivity and stable values of resistance after temperature cycling in the range $T=77-300 \mathrm{~K}$. Comparatively small changes of electrical and photoelectrical characteristics were observed in the investigated films after storage for five years under ambient laboratory conditions. The deposition method used in this investigation is characterized by high transfer efficiency of the starting material in the growth chamber (losses were less than 5\%).

\section{Experimental results and discussion}

The current-voltage characteristics were measured in the temperature range $T=196-298 \mathrm{~K}$. Shown in Fig. 1 are the current-electric field characteristics in the logarithmic scale. The solid lines represent linear $I-U$ dependences proving that the initial region of the measured characteristics obeys the Ohm law. With increase in electric field, the measured $I-U$ curves

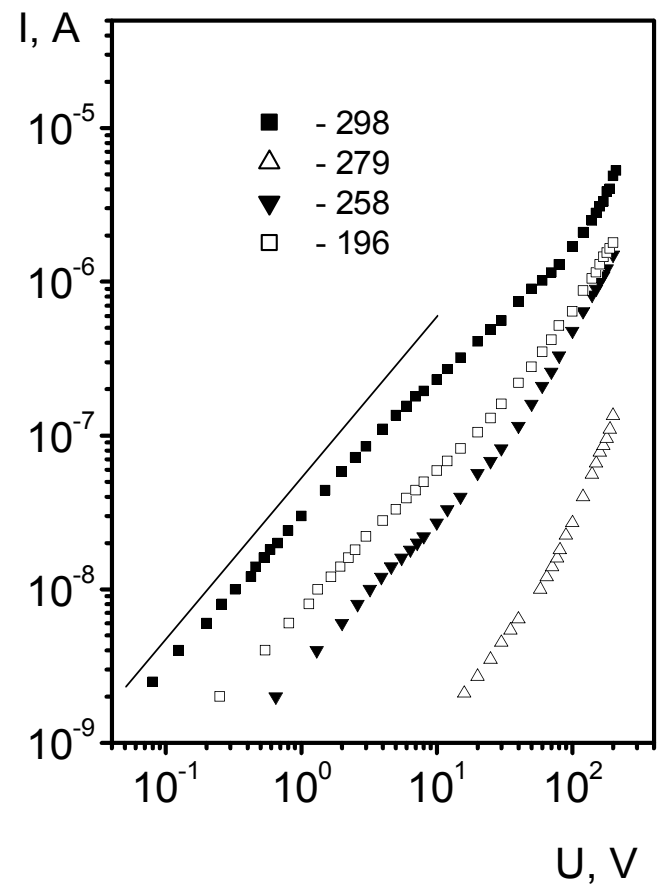

Fig. 1. Current-voltage characteristics of a polycrystalline film at different temperatures. The straight line is shown as an eye guide. 


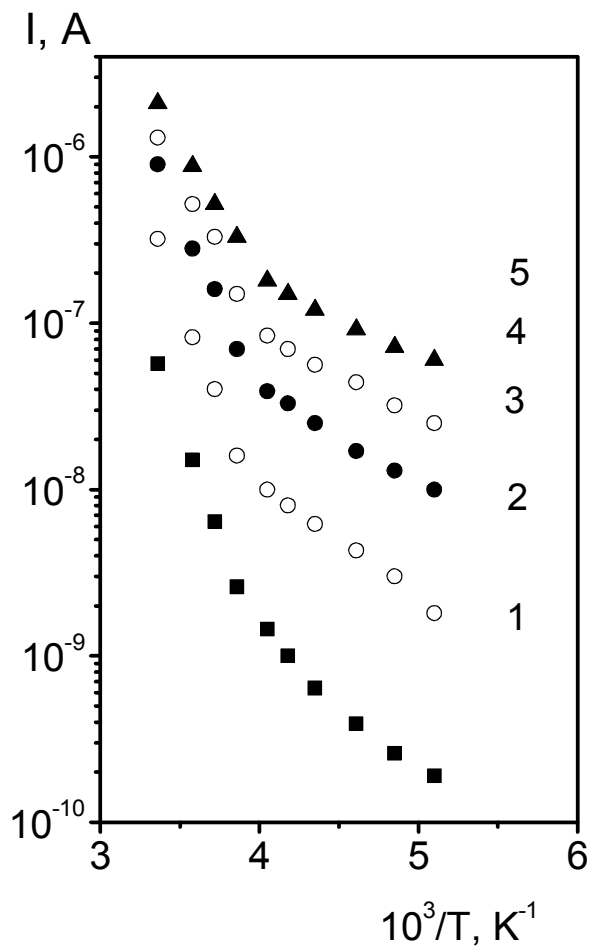

Fig. 2. Direct current vs reverse temperature dependences measured at different bias voltages, $\mathrm{V}: 1-2,2-15,3-50$, $4-80,5-120$.

start to deviate from the linear dependence. It is necessary to note that the observed non-linearity has different character for the characteristics measured at different temperatures. At room temperature $T=298 \mathrm{~K}$, they consist of the sub-linear region at lower values of the electric field followed by the super-linear one at higher values of bias voltages. To our knowledge, such $I-U$ dependence is observed in CdTe polycrystalline films for the first time. The sub-linear region is not observed on characteristics measured at low temperatures. It should be also stressed that the currentvoltage non-linearities are observed at rather low values of the electric field $F=100-300 \mathrm{~V} / \mathrm{cm}$.

The dependence of the direct current on temperature was measured at several fixed values of the electric field, Fig. 2. Experimental data are shown in the standard semilogarithmic scale, with $I$ versus $10^{3} / T$. Two temperature regions for all the measured curves can be distinguished: a sharp increase in current with temperature increasing at $T>250 \mathrm{~K}$ and a rather slow variation of current at lower temperatures. Obviously, the high temperature region corresponds to a thermally activated conduction mechanism. The activation energy of this mechanism is represented by a straight line in coordinates $\Delta E-\sqrt{F}$ (Fig. 3). The value of $\Delta E_{0}=$ $0.63 \mathrm{eV}$ at zero electric field is obtained using extrapolation procedure. Finally, Fig. 4 shows the current-electric field characteristics plotted in coordinates $I-U^{1 / 2}$. As seen, at low temperatures experimental data are well linearized within two orders of magnitude. The linearization is worse at high temperatures.

Experimental results shown in Figs 1 and 2 clearly indicate that different transport mechanisms operate in the investigated samples. The current-voltage characteristic measured at room temperature can be interpreted in terms of the grain boundary trapping theory firstly developed for silicon bicrystals [15]. The capture of mobile carriers on partially occupied trapping states at a grain boundary and subsequent rise of the barrier height results in the sub-linear current-voltage dependence. With electric field increase, these states become fully occupied, and direct current starts to increase exponentially. However, the field dependence of the direct current linear dependence of the dark current on the square root of electric field can't be explained within this theory [15]. In ordered semiconductors, these dependences are usually explained by the well-known Frenkel-Poole emission of carriers from deep-defect states in the gap [17]. As a rule, this emission is observed in the strong electric field of the order of $10^{4} \mathrm{~V} / \mathrm{cm}$. The current-voltage characteristics with a form close to the Frenkel-Poole law are also observed in amorphous and polycrystalline materials $[9,18]$. The theory of this phenomena in highly doped and compensated semiconductors has been developed by Shklovskii [18]. This theory was also used for interpretation of the dependence of conductivity on electric field in a polycrystalline semiconductor with the large-scale fluctuations of the potential barrier height [12]. However, its experimental verification in application to polycrystalline semiconductors is insufficient.

$$
\Delta \mathrm{E}(\mathrm{x} 10), \mathrm{eV}
$$

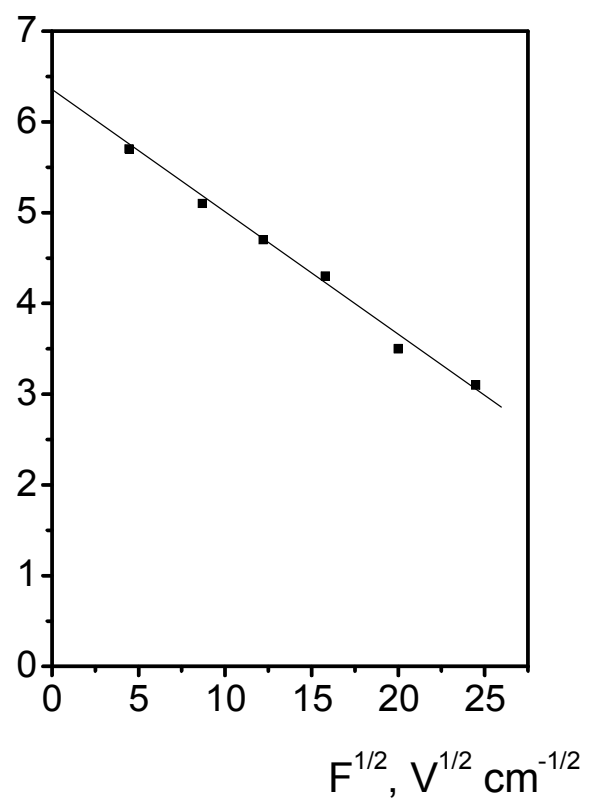

Fig. 3. Field dependence of the activation energy. 


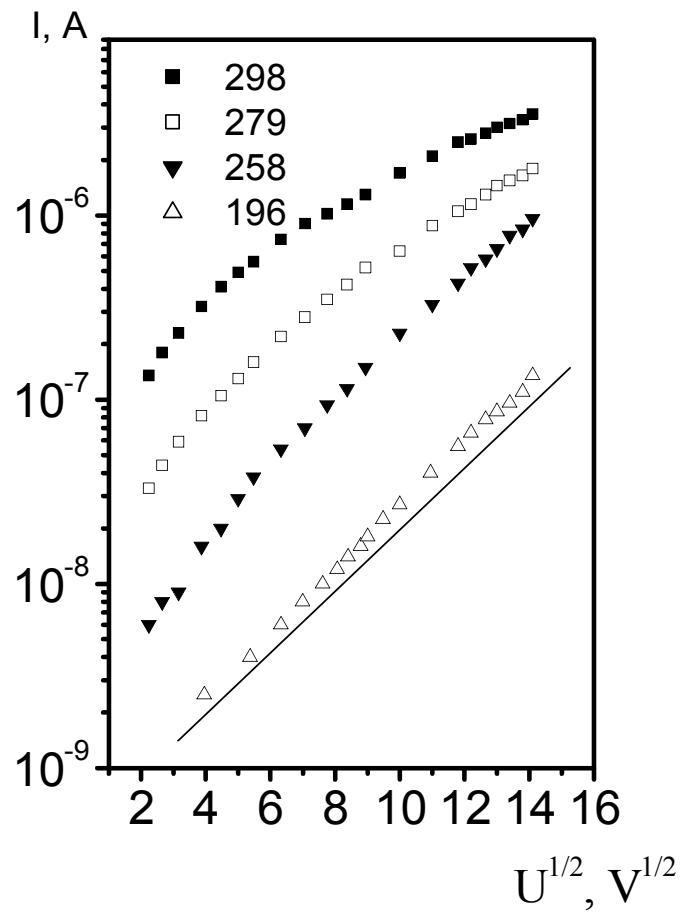

Fig. 4. Current-voltage characteristics in Schottky coordinates measured at different temperatures. The straight line represents the dependence $I-U^{1 / 2}$.

The analysis of experimental data given below is based on the Shklovskii theory [18]. The conductivity of such a semiconductor at high temperatures is determined by thermal transition of carriers on the percolation level

$\sigma=\sigma_{0} \exp \left(-\frac{\Delta E_{p}}{k T}\right)=\sigma_{0} \exp \left(-\frac{\Delta E_{p}-E_{F}}{k T}\right)$,

where $E_{p}$ is the percolation energy and $E_{F}$ is the Fermi energy. If fluctuations of potential barriers $U_{0}$ are close to $E_{p}-E_{F}$, the non-ohmic conductivity becomes apparent at rather low electric field $F$ in comparison with the ordered semiconductors:

$e F L \geq k T\left(\frac{k T}{U_{0}}\right)^{0.9} \cong k T\left(\frac{k T}{U_{0}}\right)$.

Here $L$ is the length of the large-scale fluctuations. In this case, the field dependence of the dark current has a form close to the Frenkel-Poole law

$I=I_{0} \exp \left(\frac{\beta F^{1 / 2}}{k T}\right)$,

but with a different coefficient $\beta$ in the exponent. For the Frenkel-Poole emission from deep traps in ordered semiconductors this coefficient is given by the known formula [16]

$\beta_{F P}=\left(e^{3} / \pi \varepsilon \varepsilon_{0}\right)^{1 / 2}$,
Table. Experimental and calculated material parameters.

\begin{tabular}{|c|c|c|c|c|c|c|c|}
\hline $\mathrm{K}$ & 298 & 279 & 269 & 258 & 247 & 217 & 196 \\
\hline $\begin{array}{l}\beta_{\exp }, \\
\mathrm{V}^{-1 / 2} \cdot \mathrm{cm}^{1 / 2}\end{array}$ & 0.110 & 0.125 & 0.134 & 0.145 & 0.155 & 0.166 & 0.173 \\
\hline $\begin{array}{l}\beta_{S H}, \\
\mathrm{~V}^{-1 / 2} \cdot \mathrm{cm}^{1 / 2}\end{array}$ & & & & & & 0.120 & 0.133 \\
\hline $\begin{array}{l}\beta_{F P}, \\
\mathrm{~V}^{-1 / 2} \cdot \mathrm{cm}^{1 / 2}\end{array}$ & 0.0089 & 0.0095 & 0.0099 & 0.0103 & 0.0107 & 0.0122 & 0.013 \\
\hline$\times 10^{6}$, & & 5.1 & 4.8 & 6.3 & 5.8 & 6.9 & 5.7 \\
\hline
\end{tabular}

where all symbols have usual meanings. For the Schottky emission, its value is a factor of 0.5 lower. In the Shklovskii theory, this coefficient is expressed as

$\beta_{S E}=\left(\operatorname{CreU}_{0}\right)^{1 / 2}$,

where the numerical coefficient $C$ is ranged from 0.25 to $1.0[18]$.

If percolation conductivity is dominant in the investigated films at low temperatures, the coefficient $\beta$ can be extracted from the slope of $I-U^{1 / 2}$ characteristics shown in Fig. 4. The list of experimentally determined and calculated values of $\beta$ is shown in Table. In the calculation of $\beta_{S E}$, two assumptions were made: i) the magnitude of potential barrier fluctuations $U_{0}$ is equal to $\Delta E_{0}$ and ii) the length of the large-scale fluctuations $L$ is determined for the minimal electric field values at which the non-ohmic conductivity begins. As seen from Table, there is a pronounced difference between experimental values of $\beta$ and those calculated for the Frenkel-Poole and Schottky emission. Much better coincidence is observed for the values calculated in accordance with the Shklovskii theory.

The calculated values of the length $L$ needs additional explanation. In a polycrystalline semiconductor, the distance between potential barriers are equal to the average size of grains. The physical meaning of $L$ in this case is the distance between the key barriers that determine the non-ohmic conductivity in low electric fields [11]. As seen from Table, the calculated values of $L$ are far less than the average size of grains $(\sim 10 \mu \mathrm{m})$ in the investigated films. In order to consistently explain experimental results obtained in this study, it has been assumed that the GBs in the investigated films can be treated as a highly doped and compensated semiconductor. This assumption is based on the well-known fact of a very high density of defect states $\left(>10^{18} \mathrm{~cm}^{-3}\right)$ at the GBs in CdTe polycrystalline films [19]. These high defect state densities in the GBs have major contribution to both the high density of point defects and the high degree of compensation commonly observed in cadmium telluride devices. In this case, the length $L$ has a meaning commonly used in the theory of highly doped and compensated semiconductors [18]. Lowering the potential barriers in the applied electric field shown in Fig. 3 results in emission of carriers from the electron-hole drops onto the percolation level. This 
result may be interpreted as analogue of Frenkel-Poole effect in disordered semiconductors.

As seen from Fig. 4, the percolation conduction is dominant at low temperatures. In fact, this process could be also at room temperature, but another conduction channel provided by the thermally excited movement of carriers over the potential barriers can mask it.

\section{Conclusions}

From the study of the direct current as a function of temperature and electric field the possible conduction mechanisms were established in CdTe polycrystalline films grown on glass ceramic substrates. In the low temperature region $T<250 \mathrm{~K}$, the percolation conductivity is a dominant conduction mechanism. At room temperature, the dominant conduction mechanism becomes the thermally activated process like thermionic emission or drift-diffusion transport of mobile carriers over potential barriers at the grain boundaries.

\section{References}

1. K.L. Chopra, S.R. Das, Thin-Film Solar Cells. Plenum Press, New York, 1983.

2. D.P. Holliday, J.M. Eggelston, K. Duarose, A photoluminescence study of polycrystalline thinfilm CdTe/CdS solar cells // J. Cryst. Growth 186, p. 543-549 (1998).

3. Record $16.4 \%$ efficient CdTe PV cell // III-VS Review, 14(5), p. 22 (2001).

4. D.W. Cunningham, Appolo thin film process development, in: Final Technical Report, p. 34 (2002).

5. K. Zanio, Cadmium Telluride, in: Semiconductors and Semimetals, 13, Eds. R.K. Willardson and A.C. Beer. Academ. Press, NY, 1978, p. 1-235.

6. D.V. Korbutyak, S.V. Mel'nichuk, Ye.V. Korbutyak, M.M. Borisyuk, Cadmium Impurity-Defect States and Detector's Properties. Ivan Fedorov Publ., Kyiv, 2000.

7. L.L. Kazmerski, in: Polycrystalline and Amorphous Thin Films and Devices, Ed. L.L. Kazmerski. Acad. Press, New York, 1980.
8. Polycrystalline Semiconductors. Physical Properties and Applications, Ed. G. Harbeke. Springer, Heidelberg, 1985.

9. V.B. Kvaskov, Semiconductor Devices with Bipolar Conductivity. Energoatomizdat, Moscow, 1988 (in Russian).

10. K.M. Doschanov, Mecahanism of abnormally large photovoltage in polycrystalline semiconductors // Fizika tekhnika poluprovodnikov 24(7), p. 12511258 (1990), in Russian.

11. J.H. Werner, Origin of curved Arrhenius plots for the conductivity of polycrystalline semiconductors // Solid State Phenomena 37-38, p. 213-218 (1994).

12. A.Ya. Vinnikov, A.M. Meshkov, V.N. Savushkin, Theory of non-linear percolation electroconductivity in disordered semiconductor system with intergrain barriers // Fizika tverdogo tela 24 (5), p. 1352-1359 (1982), in Russian.

13. R.P. Sharma, A.K. Shukla, A.K. Kapoor, R. Srivastava, and P.C. Mathur, Hopping conduction ip polycrystalline semiconductors // J. Appl. Phys. 57(6), p. 2026-2029 (1985).

14. V. Sosa, R. Castro, J.L. Pena, Pressure and temperature influence on CdTe thin-film deposit by close-spaced vapor transport technique // J. Vac. Sci. Technol. A 8(2), p. 979-983 (1990).

15. E.I. Goldman, A.G. Zhdan, Electrical conductivity of semiconductors with intergrain barriers // Fizika tekhnika poluprovodnikov 10(10), p. 1839 (1976), in Russian.

16. E.I. Goldman, I.B. Gulayev, A.G. Zhdan, V.B. Sandomirskii, Field characteristics of electrical conductivity in semiconductor films with intergrain barriers // Fizika tekhnika poluprovodnikov 10(11), p. 2089 (1976), in Russian.

17. A.G. Milnes, Deep Impurities in Semiconductors. Wiley, New York, 1973.

18. B.I. Shklovskii, Percolation conductivity in strong electric fields // Fizika tekhnika poluprovodnikov 13(1), p. 93 (1979), in Russian.

19. A.S. Gilmore, V. Kaydanov, T.R. Ohno, D. Grecu, and D. Rose, Impedance spectroscopy and Hall measurements on CdTe thin polycrystalline films, in: II-VI Compound Semiconductor Photovoltaic Materials, MRS Proceedings, 668 (2001). 